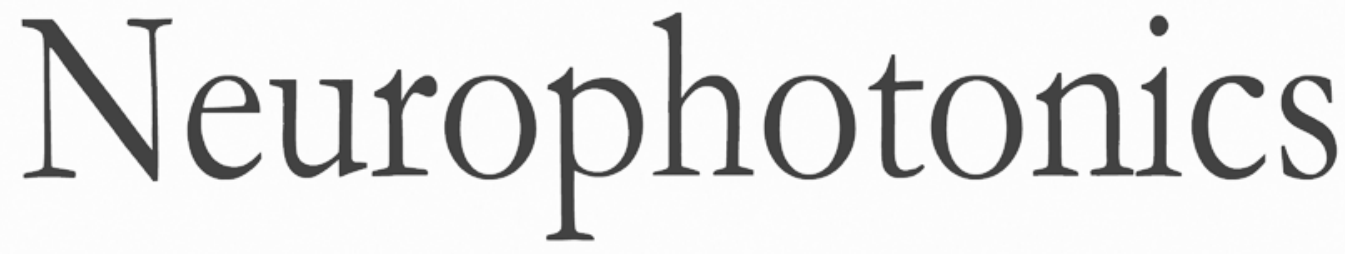

\title{
Temporal speckle-averaging of optical coherence tomography volumes for in-vivo cellular resolution neuronal and vascular retinal imaging
}

Pengfei Zhang

Eric B. Miller

Suman K. Manna

Ratheesh K. Meleppat

Edward N. Pugh, Jr.

Robert J. Zawadzki 


\title{
Temporal speckle-averaging of optical coherence tomography volumes for in-vivo cellular resolution neuronal and vascular retinal imaging
}

\author{
Pengfei Zhang, ${ }^{a}$ Eric B. Miller, ${ }^{b}$ Suman K. Manna, ${ }^{a}$ Ratheesh K. Meleppat, ${ }^{\text {a }}$ Edward N. Pugh Jr., ${ }^{\text {a,c }}$ \\ and Robert J. Zawadzki ${ }^{a, c, d, *}$ \\ aUniversity of California Davis, Department of Cell Biology and Human Anatomy, UC Davis Eye-Pod Small Animal Ocular Imaging Laboratory, \\ Davis, California, United States \\ bUniversity of California Davis, Center for Neuroscience, Davis, California, United States \\ 'University of California Davis, Department of Ophthalmology and Vision Science, Vision Science and Advanced Retinal Imaging Laboratory, \\ Sacramento, California, United States \\ ¿University of California Davis, UC Davis Eye Center, Department of Ophthalmology and Vision Science, Sacramento, California, United States
}

\begin{abstract}
It has been recently demonstrated that structures corresponding to the cell bodies of highly transparent cells in the retinal ganglion cell layer could be visualized noninvasively in the living human eye by optical coherence tomography (OCT) via temporal averaging. Inspired by this development, we explored the application of volumetric temporal averaging in mice, which are important models for studying human retinal diseases and therapeutic interventions. A general framework of temporal speckle-averaging (TSA) of OCT and optical coherence tomography angiography (OCTA) is presented and applied to mouse retinal volumetric data. Based on the image analysis, the eyes of mice under anesthesia exhibit only minor motions, corresponding to lateral displacements of a few micrometers and rotations of a fraction of $1 \mathrm{deg}$. Moreover, due to reduced eye movements under anesthesia, there is a negligible amount of motion artifacts within the volumes that need to be corrected to achieve volume coregistration. In addition, the relatively good optical quality of the mouse ocular media allows for cellular-resolution imaging without adaptive optics (AO), greatly simplifying the experimental system, making the proposed framework feasible for large studies. The TSA OCT and TSA OCTA results provide rich information about new structures previously not visualized in living mice with non-AO-OCT. The mechanism of TSA relies on improving signal-to-noise ratio as well as efficient suppression of speckle contrast due to temporal decorrelation of the speckle patterns, enabling full utilization of the high volumetric resolution offered by OCT and OCTA. ๑ The Authors. Published by SPIE under a Creative Commons Attribution 4.0 Unported License. Distribution or reproduction of this work in whole or in part requires full attribution of the original publication, including its DOI. [DOI: 10.1117/1.NPh.6.4.041105]
\end{abstract}

Keywords: optical coherence tomography; optical coherence tomography angiography; volumetric averaging; anesthetized animal; multistack registration; Fiji ImageJ.

Paper 19038SSR received Apr. 11, 2019; accepted for publication Jul. 31, 2019; published online Sep. 4, 2019.

\section{Introduction}

The retina is a visually accessible portion of the central nervous system situated at the focal plane of a natural optical system and therefore affords a unique opportunity for noninvasively visualizing the changes associated with neurodegenerative disorders and vascular alterations of neural tissue. ${ }^{1,2}$ Recent progress in high-resolution retinal imaging has achieved sufficient resolution and sensitivity to take full advantage of this opportunity in humans. ${ }^{3}$ The opportunity also applies to the mouse, a primary animal model for studying human biology and disease. ${ }^{4}$ Mouse models are important not only in basic vision science and ophthalmic research, ${ }^{5}$ including studies of the mechanism of phototransduction, ${ }^{6,7}$ but also in general neuroscience. ${ }^{8}$ Interestingly, the special optical features of the mouse eye, which has a numerical aperture (NA) twice than that of the human eye, ${ }^{9}$ offer a potentially higher resolution for retinal imaging. ${ }^{10}$ However, the retinal neurons anterior to photoreceptors-i.e., bipolar, horizontal, amacrine, or ganglion cells (GCs) - are nearly transparent, making them very difficult to image in

*Address all correspondence to Robert J. Zawadzki, E-mail: rjzawadzki@ ucdavis.edu the living eye using imaging modalities employing only labelfree backscattered light.

Scanning laser ophthalmoscopy (SLO) ${ }^{11}$ and optical coherence tomography (OCT) $)^{12}$ are two complementary in-vivo retinal imaging modalities. ${ }^{13-16}$ Both detect backscattered light, and SLO can also detect fluorescence. SLO and OCT have been successful in imaging the photoreceptor mosaic and retinal microvasculature with adaptive optics (AO), ${ }^{17,18}$ but only very recently there have been reports of imaging the highly transparent cells in the inner retina. Rossi et al. ${ }^{19}$ reported imaging GC somas visualized by AO-SLO, implementing aperture split detection; Liu et al. $^{20}$ and Wells-Gray et al. ${ }^{21}$ reported visualization of GC somas using AO-OCT with volume averaging. Interestingly, AO-OCT with volume averaging produced images of GCs with unprecedented clarity, allowing reliable mapping of morphometry of retinal neuron somas across the living human retina. ${ }^{20}$ There have been many reports of imaging inner retinal neurons in the mouse retina labeled by extrinsic fluorescent proteins or dyes and detected by fluorescence emission SLO. ${ }^{13}$ There have also been reports of imaging retinal neuron somas using AOSLO with split detection like in human studies. ${ }^{22}$ However, there have been no reports on the visualization of inner retinal neurons based on volume averaging of OCT or AO-OCT data from the mouse eye. Considering the important role of the mouse model 
in biomedical research, the ability to visualize these cells is clearly desirable.

In this paper, we present a general framework developed in our laboratory which uses mouse retinal OCT/optical coherence tomography angiography (OCTA) to achieve high-contrast volumetric images of inner retinal neurons (with OCT) and corresponding microvasculature (with OCTA). After registration and application of temporal speckle-averaging (TSA) method, cellular-level resolution volumetric neurovascular retinal mapping is achieved. The key to successful TSA lies in using an OCT/OCTA volume registration method that corrects for mouse eye movement occuring during imaging under general anesthesia. We develop a stepwise method to register a series of successively acquired three-dimensional (3-D) OCT volumes using three consecutive two-dimensional (2-D) registrations with reliable and robust registration tools available in ImageJ. ${ }^{23} \mathrm{~B}$-scan and en face images from both OCT intensity and OCTA ${ }^{24,25}$ volumes are directly compared side by side to illustrate the effect of speckle-averaging, which is further quantified by various image metrics and analyses. Finally, two examples, one reconstructed from 150-OCT-volumes of a pigmented $(\mathrm{C} 57 \mathrm{Bl} / 6 \mathrm{~J})$ mouse and the other from a 50-OCTA-volumes of a B6 albino mouse, are presented. Two important retinal structures, retinal neuron somas and the choriocapillaris, are visualized for the first time in the living mice without extrinsic contrast agents.

\section{Methods}

Unlike in humans, in-vivo animal retinal imaging is often performed under anesthesia to reduce unnecessary stress. Common problems associated with long-term imaging of anesthetized animals include tear film breakup, drying of the corneas, and development of cold cataracts. These effects can be overcome by the use of a contact lens and transparent gel to maintain the cornea clear and moist, ${ }^{13,26}$ and by maintaining and monitoring body temperature during imaging. Most eye movements, including involuntary drift and saccades, are not present in mice under anesthesia. ${ }^{27}$ Despite the reduction in eye movements, some eye motion, caused by animal breathing and consequent head movements, occurs with consequent image artifacts during retinal imaging. We observed that these residual motions are mostly small (with noted exceptions) and give rise to distortions that can be characterized as rigid-body displacements between volumes, which can be corrected with a simple and robust stepwise correction method employing three serial 2-D registrations in two separate image planes.

\subsection{Mouse Positioning and Anesthesia}

All mice husbandry and handling were in accordance with protocols approved by the University of California Animal Care and Use Committee, which strictly adheres to all National Institutes of Health (NIH) guidelines and satisfies the Association for Research in Vision and Ophthalmology guidelines for animal use. Adult pigmented C57BL/6J and B6 albino mice were obtained from Jackson Laboratories and maintained on a 12:12, 100-lux light cycle. During the experiments, mice were anesthetized with the inhalational anesthetic isoflurane $\left(2 \%\right.$ in $\left.\mathrm{O}_{2}\right)$, and their pupils were dilated with medical grade tropicamide and phenylephrine. A contact lens and gel (GelTeal Tears, Alcon) were used to maintain the cornea transparency for in-vivo retinal imaging. ${ }^{26,28}$ A commercially available bite bar (Kopf Instruments, Inc., Model 1923-B Mouse Gas
Anesthesia Head Holder) was used to stabilize the mouse head and deliver the oxygen and isoflurane.

\subsection{University of California Davis Multimodal SLO/OCT Mouse Retinal Imaging System}

A combined SLO/OCT system was used for imaging the mouse retina. ${ }^{13}$ The beam at the mouse pupil was $0.5 \mathrm{~mm}$, which corresponds to a NA of 0.12 for the imaging system. ${ }^{29}$ The light power is $100 \mu \mathrm{W}$ for SLO (488 nm laser, Coherent) and $600 \mu \mathrm{W}$ for OCT (center wavelength $860 \mathrm{~nm}$ with a bandwidth of $132 \mathrm{~nm}$, SLD, Superlum Ltd.). A custom spectrometer using Balser line scan camera with 2048 pixels was used to acquire the OCT spectra. OCT spectra were acquired at a $100-\mathrm{kHz}$ A-scan rate using custom Labview software that can operate in continuous volume acquisition mode. The volume acquisition time was $\sim 3$ s for OCT (512 A-scans/B-scan, 512 B-scans/volumedata in Fig. 6) and $\sim 6 \mathrm{~s}$ for OCTA (540A-scans/B-scan, 1080 B-scans/volume-data in Fig. 7). The OCT volumes were saved at 5 volumes $/ \min \left(\Delta T_{\mathrm{OCT}}=12 \mathrm{~s}\right)$, and OCTA volumes were acquired at 2.5 volumes $/ \min \left(\Delta T_{\text {OCTA }}=24 \mathrm{~s}\right)$.

Initial postprocessing was implemented by custom Matlab ${ }^{\mathrm{TM}}$ code implementing standard OCT processing, including direct current subtraction, wavelength-to- $k$ (wave number) resampling and interpolation, dispersion compensation, Hann windowing, and FFT. ${ }^{30,31}$ The OCTA data were processed by a custom phase-variance OCT (pv-OCT) $\mathrm{C}++$ code to visualize blood flow and generate OCTA B-scans. ${ }^{32,33}$ The serially recorded volumetric data sets, including intensity and OCTA data, were further coregistered to correct for motion artifacts and allow averaging.

The serial volume registration was implemented by image processing in MATLAB and ImageJ. As described in detail below, serially collected volumetric data set were initially processed with the custom MATLAB code for registration, and then the ImageJ "MultiStackReg" and "StackReg" plugins were applied to coregister prearranged series of stacks.

\subsection{Retinal Image Movements in Anesthetized Mice during Imaging}

Although the general anesthesia used during retinal imaging greatly helps to reduce eye movements that distort images, the remaining eye movements are not negligible, especially for long acquisition times if cellular resolution averaging is desired. Here we present a simple observation of the retinal movements that occur in anesthetized animals and their effects on volumetric image data. Figure 1(a) shows the Cartesian coordinate system on the retina used to represent the 3-D data. Here the $x$ and $y$ axes correspond to OCT galvo-scanners' fast and slow scanning directions, respectively, while the $z$ axis is the OCT depth or A-scan direction (along which data are acquired within the pixel dwell time of $10 \mu \mathrm{s})$. The fast OCT $x$-scanner, which generates B-scans, scans at $\sim 200 \mathrm{~Hz}$, while the slower $y$-scanner, which shifts the B-scans to generate the volumes, sweeps across the image field at $\sim 0.3 \mathrm{~Hz}$. Figure 1(b) depicts the most common image displacements that we observe. Slower motion artifacts that arise from small eye drift result in translation $(x, y, z)$ and rotation $(\varphi, \theta)$ between successive volumes, and can be easily corrected by rigid-body transformation using ImageJ stack registration. We previously used a similar framework to correct for motion artifacts within a single volume in human retinal imaging with $\mathrm{AO}-\mathrm{OCT},{ }^{34}$ but here we apply a 
(a)

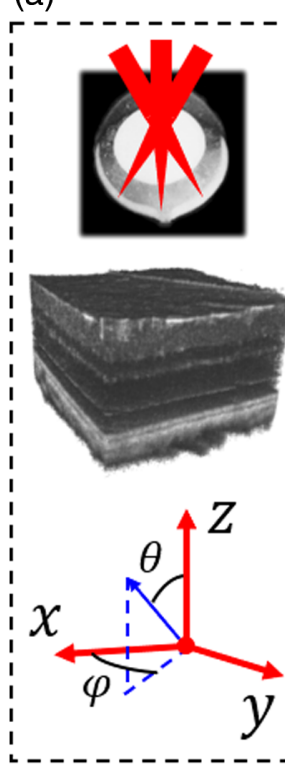

(b)

Common distortion errors

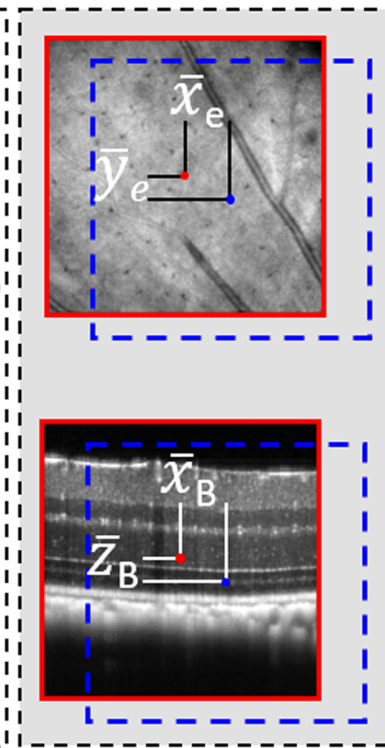

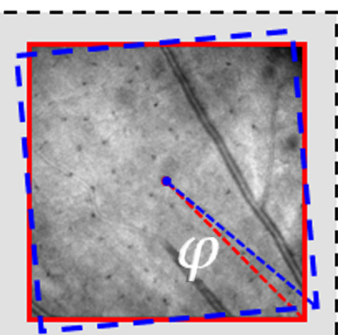

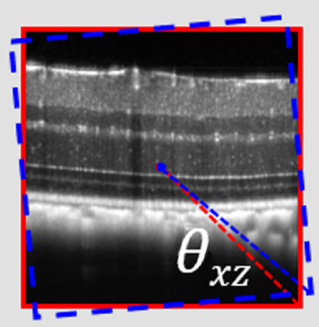

(c) Rare distortion errors

(d) Mouse breath and intraframe distortion

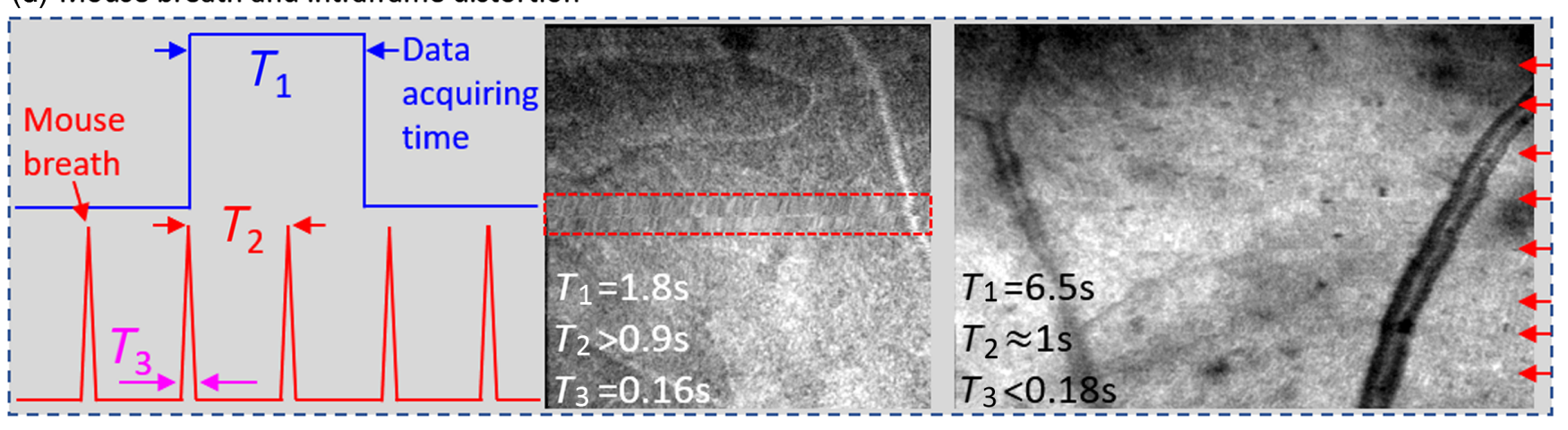

Fig. 1 Characterization of mouse eye movements during retinal imaging under anesthesia. (a) Schematic representation of the in vivo retina scanning through the eye's optics (top panel); although the retina is a curved surface, the OCT volume data are flat for modest scan angles $(<30 \mathrm{deg})$ (middle panel), and so can be displayed in a 3-D Cartesian coordinate system. For such a 3-D volume, there are 6 degrees-of-freedom of movement, taken to be translations along the three axes $(\bar{x}, \bar{y}$, and $\bar{z})$ and the corresponding rotations (bottom panel). (b) Visual representation of the most common distortions observed between OCT data sets: lateral displacements $\bar{x}_{e}$ and $\bar{y}_{e}$, and rotation $\varphi$ in the $x-y$ plane; with respect to B-scans, the errors from consecutive volumes are displacements, $\bar{x}_{B}$ and $\bar{z}_{B}$ and rotation $\theta$ (for simplicity we depict rotation only in the $x$ and $y$ planes). All can be corrected efficiently by rigid-body transformations. (c) Other potential errors include rotations along the $x / y$ axis resulting in nonuniform scaling in $x$ and $y\left(x^{\prime}\right.$ and $y^{\prime}$, respectively) and overall scale changes (magnification); both can be corrected by affine transformations. (d) The mouse eye movement caused by breathing is much shorter than the interval between breaths. Thus, the corresponding distortion is relatively small and does not affect registration between en face images. Left panel shows timing of data acquisition (blue) with frame acquisition time $\left(T_{1}\right)$, and mouse breathing (red) characterized by time interval between breaths $\left(T_{2}\right)$ and duration of breathing artifacts $\left(T_{3}\right)$; middle and right panels show two examples of OCT single volume en face projections for short (middle panel) and long (right panel) volume acquisition times indicated by $T_{1}$. Locations of breathing artifacts are marked by a dashed rectangle in the middle panel and arrows in the right panel, respectively.

multistep process to achieve volume-to-volume coregistration. In addition to the relatively small volume displacements just described, there are also rarely occurring distortions that create scaling changes between volumes [Fig. 1(c)]; these rare distortions are produced by rotations of the eye along the $x$ or $y$ axis or axial displacement of the eye as a whole. Unlike in most human retinal imaging, a contact lens and gels were used in our mouse retinal imaging system, which resulted in the creation of more degrees of freedom for image distortions. For example, moving the contact lens $1 \mathrm{~mm}$ away from the cornea (resulting in gel thickness changes) can easily increase the scaling factor to $30 \% .{ }^{26}$ If the gel thickness changes unevenly across the pupil (e.g., during eye rotation along $x$ or $y$ axis one side becomes thicker than the other side), nonisotropic scaling between $x$ and $y$ axes will occur. Although such displacements are rare, they can nonetheless be detected in the image analysis and corrected by the ImageJ affine transformation. However, based on our experience the most efficient approach is to examine registered volumes using en face intensity projection after first correcting them for the common motion artifacts, and simply discard these 
volumes whose en face projections show scaling deformations, rather than to use more complex registration. The interval between mouse breaths can be $\sim 1$ to $3 \mathrm{~s}$, and the eye movements caused by the mouse breathing will be recorded during the OCT volume acquisition [Fig. 1(d)]. We noticed that the duration of the eye movement during breathing is much shorter than the interval between breaths; thus, only small portion of the data is distorted [Fig. 1(d) middle and left panels]. These distortions do not affect the registration between en face images and are not noticeable after averaging. Thus, the image distortions caused by mouse breathing were ignored in our method.

\subsection{Correction of the Mouse Retinal Movements Present during Anesthesia Using Stepwise OCT/OCTA Volume Registration}

To correct the retinal motion artifacts described above, a method of image registration between consecutive volumes is needed. However, no broadly accepted serial volume registration is commonly available. Thus, custom volume registration software had to be developed. In developing, we tried to use well vetted and simple freely available registration tools. We found that one of the simplest and most reliable 2-D image alignment tools in ImageJ-StackReg plugin ${ }^{35}$ - and its extensions such as MultiStackReg ${ }^{36}$ work very well for registering successive B-scans, as well as en face projections of OCT Volumes.
Notably, the MultiStackReg plugin aligns stacks of images to one single reference image, thereby eliminating the error accumulation that occurs with the StackReg plugin. Thus, we implemented a stepwise 2-D registration for serial OCT/OCTA volume registration. Importantly, when several imaging channels are acquired simultaneously (e.g., OCT intensity and OCTA), one needs to be able to align the data to one of the channels only (e.g., OCT intensity) and then apply the correcting transformations directly to the other channels. This important feature is also available in MultiStackReg plugin.

We now describe the stepwise volume registration method, which uses a series of 2-D registrations to correct the motion artifacts between volumes (Fig. 2). After acquisition of a series of OCT volumes, for each OCT volume we first create an en face intensity projection image from the whole volume or, in case when the vascular features are not visible enough, projection from the inner retina [from outer plexiform layer (OPL) to nerve fiber layer (NFL)] was used to improve the overall registration performance. Then we use the MultiStackReg ImageJ plugin to register the full set of en face images to a reference image from the middle of the series. Because the intravolume distortion only occupies a small portion of the data, the single middle frame (in the middle of the time series stack) is usually sufficient to be selected as a proper reference. The transformation matrix for the registration of each en face image was saved, and then applied to the entire volumes in the OCT series (a)

\section{Multivolumes acquisition}

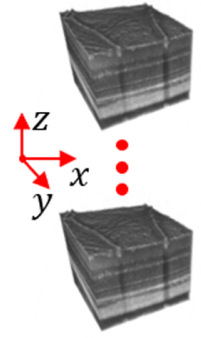

(b) face images Transformation matrix

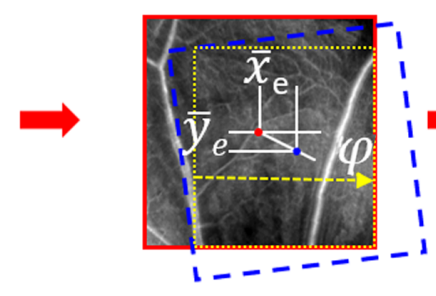

Apply to volumes (intensity

(c) and other channels)

(d)
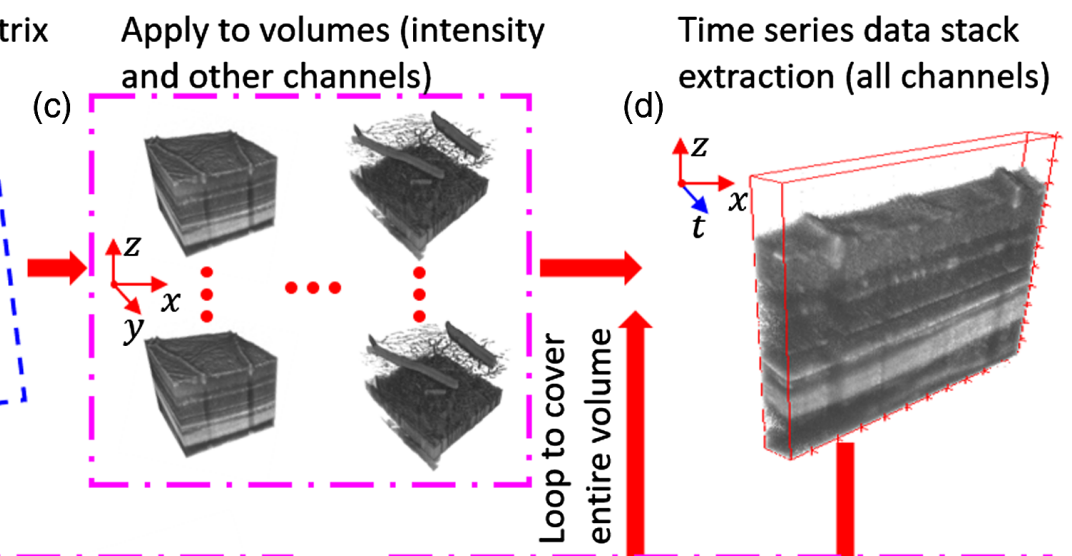

(g)

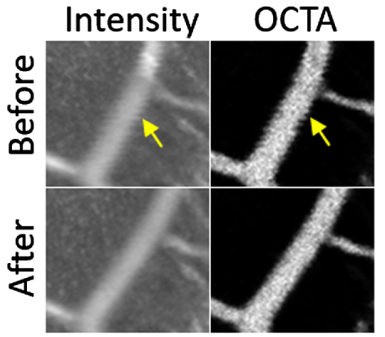

Before and after final stack registrations

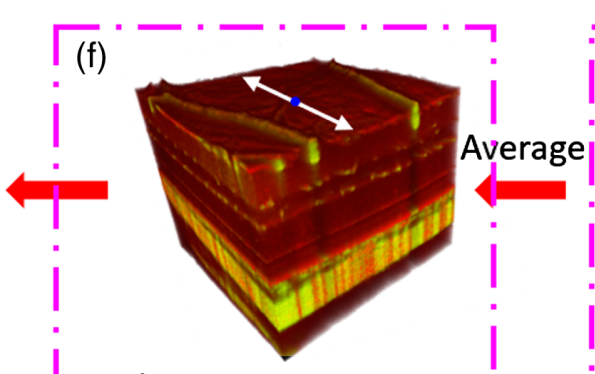

Stack registration between averaged B-scans

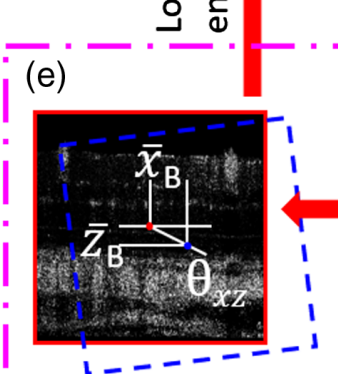

Apply transformation Time series B-scan to other channels stack registration

Fig. 2 Flowchart for stepwise multivolume registration. (a) Acquisition of multiple OCT volumes. (b) Extraction of a transformation matrix characterizing motion between volumes using en face OCT intensity projection images from each volume. (c) Application of the transformation derived in step (b) to shift/rotate all data in the multichannel volumes. (d) Visualization of a representative time series stack (BM stack) of OCT intensity data for one $y$ location. (e) The set of B-scans comprising the BM stack for each $y$ position is registered, correcting for transformations between scans in the stack. (f) TSA intensity (red) and OCTA (green) volumes are created by directly averaging for fine alignment. (g) En face images illustrating the effect of a final stack registration of TSA B-scans with the StackReg plugin, which corrects for fine motion artifacts between adjacent B-scans in the TSA volume. 
(including the intensity and other channels, e.g., OCTA). After registration and motion correction with the transformations, the volumes were next cropped to maintain only the overlapping regions, as shown by the smallest rectangle in Fig. 2(b); this removes empty regions to allow efficient second step of registration between the B-scans. In the next step, a temporal series of B-scans from the same $y$ location in the series of OCT volumes was extracted and enclosed in a stack for registration (BM stack). Then, the motion errors between successive B-scans in the BM stack were corrected with MultiStackReg and the transformations were recorded; the same transformation matrices were then applied to all other channels of the data sets (e.g., OCTA). Specifically, this latter step must be performed for each B-scan within the serial OCT volumes [steps in Figs. 2(d)-2(e)] until the entire overlapping volume region has been corrected. After BM-stack registration, we then averaged the series of B-scans from each $y$ location to achieve the TSA volume.

However, it was noted that in the TSA volumes there were sometimes slight offsets (possibly arise from intravolume motions caused by mouse heartbeat or scanners off-axis vibration) between adjacent B-scans [as shown in Fig. 2(g) top image, arrows]. Usually, a simple registration using StackReg plugin with intensity OCT data assigned to one color (e.g., red) channel and OCTA information assigned to another color (e.g., green) channel was performed to correct for the fine position errors between adjacent TSA B-scans. This allowed the production of a smoothed TSA volume of OCT intensity and/or OCTA data. The core of this method relies on three serially performed registrations, marked by three dash-dotted rectangles in Fig. 2.

\subsection{Effects of TSA on OCT Data Sets}

The average volume created by TSA can be expected to provide improved visualization of weakly scattering structures in the 3-D sample volume on the basis of general principles. For example, if backscattering from a structure spanning several adjacent voxels results in an average of $\bar{n}$ photons collected by the detector, Poisson fluctuations of magnitude $\sqrt{\bar{n}}$ from voxel to voxel could make the structure undetectable in a single volume. TSA with $N$ volumes would reduce the fluctuations to $\sqrt{\bar{n} / N}$, while the signal from all voxels remains $\bar{n}$, so that TSA results in increased sensitivity and dynamic range of OCT intensity images. Averaging without the precise alignment provided by TSA, however, would not confer these benefits because the nominally identical voxel in a series of poorly aligned volumes would have variations arising from sample-to-sample variation in the underlying structure due to motion. What would result in a reduction of both resolution and speckle contrast? Interestingly, averaging without the precise 3-D alignment provided by TSA, but with the simple 2-D BM-scan registration as it is done in most commercial clinical OCT systems, still confers most of the TSA benefits. This is due to the fact that even imperfectly coregistered B-scans in $y$ axis (slow scanning direction) have nominally similar voxel values in the same retinal layer. This allows achieving speckle contrast reduction and signal-to-noise ratio (SNR) benefit of averaging but at the cost of a reduction in lateral resolution. ${ }^{37}$

There are additional benefits of TSA that goes beyond this classical improvement in image sensitivity and dynamic range. One arises from improved phase stability associated with each voxel: this increases the fidelity of the OCTA data set. ${ }^{24}$ A second is a visible reduction of speckle contrast, which greatly improves the visibility of cellular and subcellular features on OCT intensity data. ${ }^{38}$ To quantify these benefits, we calculated cross correlations between intensity BM stacks to estimate the time needed for full decorrelation of the speckle fields. We also measured changes in speckle contrast and intensity histograms for TSA performed on a large number $(N=150)$ of volumes. Here, we introduce three parameters that are used to quantify changes in speckle contrast.

\subsubsection{Contrast-to-noise ratio}

To estimate both effects of TSA, the improvement in SNR, and reduction in speckle contrast, we use the contrast-to-noise ratio (CNR). Here the intensity from a region of interest (ROI) from the retina was assigned as a signal, and a background area from the vitreous was defined as the background (noise). ${ }^{39}$ Then, the CNR was calculated as

$\mathrm{CNR}=\frac{\bar{I}_{\mathrm{ROI}}-\bar{I}_{\mathrm{bg}}}{\sqrt{\left(\sigma_{\mathrm{ROI}}^{2}+\sigma_{\mathrm{bg}}^{2}\right) / 2}}$,

where $\bar{I}_{\text {ROI }}$ and $\bar{I}_{\text {bg }}$ are the mean values of the intensity within an ROI and background regions, respectively, and $\sigma_{\mathrm{ROI}}$ and $\sigma_{\mathrm{bg}}$ are their corresponding standard deviations (SDs).

\subsubsection{Normalized speckle contrast}

Normalized speckle contrast (NSC) was used to quantify the speckle contrast suppression effect due to temporal averaging. NSC is defined as

$\mathrm{NSC}=\frac{\sigma_{I}}{\bar{I}}=\frac{\sigma_{\mathrm{ROI}}}{\bar{I}}$,

where $\sigma_{I}$ is the SD of the image intensity $(I)$ in a given region and $\bar{I}$ is its average. ${ }^{40}$ The averaging of independent speckle patterns is widely understood to be the primary mechanism by which speckle is reduced. If in a depth-restricted portion of a B-scan, the intensity of all pixels is independent and identically distributed, and $M$ uncorrelated speckle patterns are averaged, the speckle contrast of the average will obey the following general relation: ${ }^{40}$

$$
\frac{\mathrm{NSC}_{M}}{\mathrm{NSC}_{1}}=\left(\frac{\sigma_{I}}{\bar{I}}\right)_{M} /\left(\frac{\sigma_{I}}{\bar{I}}\right)_{1}=\frac{1}{\sqrt{M}} .
$$

While this effect is related to the general effect of averaging described above, it is distinctive, because speckle contrast is voxel-to-voxel variation arising from scattering entities smaller than the 3 -D point-spread function (PSF). ${ }^{41}$ The scan-to-scan variation in speckle underlying Eq. (2) would arise if the number of scatterers in each PSF volume or their mutual interference fluctuated from volume to volume about the same mean value. Here we assume that the variance in intensity arising from speckle is much higher than the one arising from the noise.

\subsubsection{Effective number of uncorrelated speckle patterns $(m)$}

Actually, the mean intensity of speckle from voxels at different locations will vary in a sample. In this case, the speckle intensity distribution can be described as a case of the full 
$K$-distribution. ${ }^{42,43}$ This distribution has a parameter $(m)$ that can be used to estimate the number of independent speckle patterns: ${ }^{42}$

$$
\begin{aligned}
\mathrm{p}_{K}\left(I_{\mathrm{sp}} \mid \alpha, \mu, m\right)= & \frac{2}{\Gamma(\alpha) \Gamma(m)} I_{\mathrm{sp}}^{\frac{\alpha+m-2}{2}}\left(\frac{\alpha m}{\mu}\right)^{\frac{\alpha+m}{2}} \\
& \times K_{\alpha-m}\left(2 \sqrt{\frac{I_{\mathrm{sp}} m \alpha}{\mu}}\right),
\end{aligned}
$$

where $I_{\mathrm{sp}}$ represents the possible values of intensities in the fully developed speckle pattern. Here $\alpha$ and $\mu$ are the shape parameter and expected value, respectively, of the local mean intensity variation (assumed to follow a gamma distribution), $K_{\alpha}(x)$ is the modified Bessel function of the second kind with order $\alpha$, and $m$ is the number of uncorrelated speckle patterns. ${ }^{42}$ We fitted OCT intensity histograms of TSA scans in the BM stack with this function to estimate the number of uncorrelated speckle patterns in the original temporal series.

\section{Results}

In this section, we provide the results of our analysis of mouse eye movements during anesthesia, followed by a comparison between single and TSA OCT intensity and OCTA data sets. Then we quantify the effect of TSA on CNR improvement and speckle contrast reduction. Finally, examples of applying TSA to OCT volumes of pigmented $(\mathrm{C} 57 \mathrm{~B} 1 / 6 \mathrm{~J})$ and $\mathrm{B} 6$ albino mice are provided.

\subsection{Experimental Analysis of Mouse Retinal Movements during Serial Volume Acquisition}

To characterize the motion artifacts (described above, Fig. 1) that underlie motions in the images of anesthetized mice, we acquired and subsequently analyzed a multimodal volumetric data set centered on a single fluorescent retinal ganglion cell (RGC) (Fig. 3). This thy1-YFP-16 transgenic mouse (The Jackson Laboratory) expresses a yellow fluorescent protein

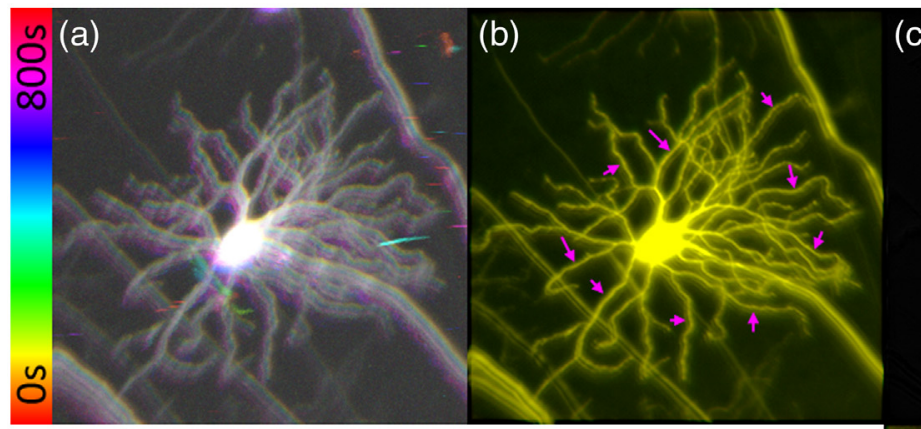

(c)
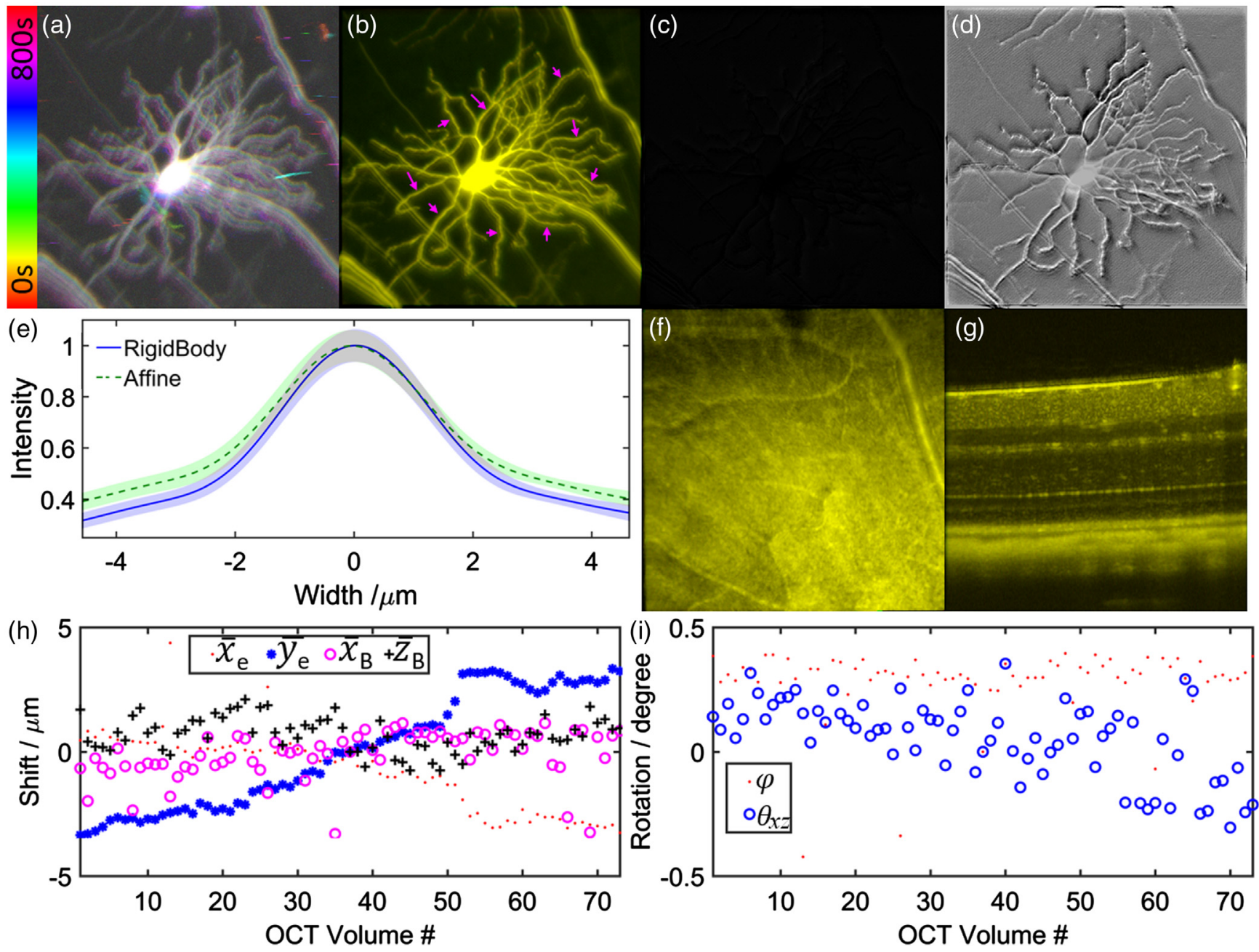

Fig. 3 Visualization of motion artifacts present during serial mouse retinal imaging, for over $800 \mathrm{~s}$, and their correction. (a) A temporally color-coded fluorescent SLO image of a retinal GC expressing YFP. (b) Average composited RGC image created by placing the results from rigid-body and affine registrations into red and green channels, respectively. (c) Difference between grayscale intensity images obtained with two different registration methods. (d) The same image displayed using ImageJ 32-bit operation of subtraction with stretched intensity. (e) Comparison of dendrites' line profiles extracted from averaged images obtained using two different methods; dendrites locations were marked in (b) with arrows. Errors represent standard error of the mean (SEM). (f), (g) Merged OCT en face, and B-scan generated the same way as (b). (h), (i) Translational and rotational errors extracted from the registrations of (f) and (g). 
(YFP) at high levels in motor and sensory neurons, as well as in subsets of central neurons. The complex, unique pattern of the RGC's submicrometer fluorescent dendrites provides an ideal target for examining motions over an extended imaging session. During the $\sim 14$ - min imaging session, 400 consecutive SLO images and 73 consecutive OCT volumes were simultaneously collected at the same retina location, which scanned $\sim 300 \times 300 \mu \mathrm{m}^{2}$ with $400 \times 400$ pixels sampling. To illustrate the effect of motion between volumes, Fig. 3(a) displays the SLO fluorescence images of a single RGC color-coded by time: motions are manifest as blurring of the image and color fringes. Figure 3(b) shows the result of combining the two images after assigning them with red and green for rigid body and affine registration images, respectively. The improvement in sharpness reveals that these registrations have removed much of the motions of the unregistered average image [Fig. 3(a)], while the uniform color of the combined image indicates that there was little difference between corrections obtained with the two registration methods. Figure 3(c) shows an image of the absolute value of the pixel-by-pixel difference between the images obtained with two different registrations, further revealing that there are no major differences. Nonetheless, a contrast-stretched 32-bit difference between them reveals a small residual difference [Fig. 3(d)]. The pseudo-3-D relief appearance of the cell dendrites is due to slightly sharper and brighter local fluorescence after registration by rigid-body transformation as compared to that with affine. Figure 3(e) provides the averaged line profiles from nine different locations across the GC dendrites [Fig. 3(b) arrows] extracted from two averaged images, one obtained by the rigid body and one by affine registrations. Note that rigid-body registration creates a sharper line profile. This result also suggests that the shadow near the dendrites seen in Fig. 3(d) is mainly caused by the difference in sharpness and brightness, and not by a lateral mismatch between two averaged images. This comparison also reveals that there is a negligible scaling transformation [Fig. 1(c)], because rigid-body correction (no scaling correction) works better than affine correction (correction with scaling transformation). Figures $3(\mathrm{f})-3(\mathrm{~g})$ show the result of coregistration and correction of OCT volumes using the same visualization method as in Fig. 3(b) but applied to the OCT en face and BM stack, respectively. No visible differences (colors) on en face intensity projection and B-scan can be seen. This confirms that the rigid-body registration is sufficient to correct for retinal motion artifacts present in serial volumetric OCT data sets acquired in anesthetized animals. Finally, Figs. 3(h)-3(i) shows translational and rotational errors extracted from the transformations to generate Figs. 3(f) $-3(\mathrm{~g})$. Overall, the positional variation of the mouse retina during this 14-min imaging session had a range of $\pm 5 \mu \mathrm{m}$ and rotation variation of $\pm 0.3 \mathrm{deg}$. In summary, the analysis of this multimodal volumetric data set reveals the nature of the motions that occur in an imaging session and shows that the method developed corrects them successfully.

\subsection{Direct Comparison of Images from Single and Temporal Speckle-Averaged OCT Volumes}

Because OCT involves the interference of partially coherent light, OCT images necessarily have high speckle contrast, which arises from scattering particles in the sample that are smaller than the 3-D PSF. ${ }^{41}$ Speckle contrast degrades image quality, making it impossible to resolve fine structures near diffraction-limited resolution. Despite its spatially random appearance, the speckle pattern of a nonvarying sample is fully determined by the underlying spatial distribution of scatterers and would not vary from scan to scan if nothing in the sample moves. However, in-vivo sub-PSF scale scattering particles (e.g., mitochondria and other organelles) are not static, resulting in variation in the speckle pattern between consecutive volumes. ${ }^{44}$ At the same time, larger morphological structures such as cell bodies and vasculature are static on the timescales of image acquisition, allowing image registration. As a consequence, averaging of serial volumes reduces speckle contrast, while maintaining structural information from objects larger than the PSF, yielding images with improved SNR and image contrast. Comparison of single and TSA volumes provides a means of visualizing the speckle-reducing effect of averaging (Fig. 4). Figure 4 shows the comparison between single OCT and OCTA volume and TSA OCT and OCTA volumes (from 150 and 50 serially acquired OCT and OCTA volumes, respectively). In the TSA OCT B-scan, one can distinguish the walls of two large blood vessels in the NFL (arrows). The TSA OCT en face image clearly shows much better blood vessel contrast (including blood vessel walls, arrows), nerve fiber bundles, and novel structures resembling cell somas (arrowhead).

TSA also greatly improves OCTA data. A comparison between a single volume and TSA OCTA is shown in Figs. 4(e)-4(h), where even smallest capillaries are clearly visible in the OCTA B-scan (red arrows). Similarly, the en face projection of capillaries from OPL shows great improvement of signal (capillaries)-to-noise (random noise between capillaries) ratio. For example, even some parts of the capillaries hidden in the shadows of larger NFL blood vessels in single volumes appear continuous (arrows) in the TSA volume.

\subsection{Quantification of the Effect of Temporal Speckle-Averaging}

As described above, the improved cellular contrast offered by TSA relies on averaging uncorrelated speckle patterns to suppress the speckle contrast and improve the SNR of resulting images, what can be quantified by CNR. To estimate the effect of TSA, we analyzed OCT intensity data from the experiment, as shown in Figs. 4(a)-4(d). First, we calculated the cross correlation between B-scans from the BM stack as a function of the time interval between scans [Fig. 5(a)]: the correlation is seen to decline to about 0.2 over $120 \mathrm{~s}$ (10 volume scans). Figure 5(b) shows the average ( \pm SEM) correlations calculated for 15 pairs of B-scans separated by different times for three different retinal locations with the fitted cross-correlation curves matching the rectangles depicting corresponding retinal locations in Fig. 4(a): while generally true that the longer time interval between two B-scans, the lower the correlation, the results suggest that the maximum decorrelation is achieved in about $2 \mathrm{~min}$. While this implies that waiting 2 min between scans might be optimum for reducing speckle, it needs to be kept in mind that TSA of volumes obtained more frequently, nonetheless, improves the visibility of weakly scattering structures according to $1 / \sqrt{N}$, as discussed above. This decrease in the noise of the background $\sigma_{\text {bg }}$ as a function of a number of averaged volumes, $N$, is shown in Fig. 5(c). However, the actual data visibility defined by CNR in Eq. (1) exhibits mostly dependence on NSC. NSC [Eq. (2)] declines with increasing $N$, but as expected this decline does not follow the simple relation [Eq. (3)], showing that the number of independent speckle patterns $M$ is less than $N$ for this volume acquisition rate [Fig. 5(d)]. The combined effect of speckle- 

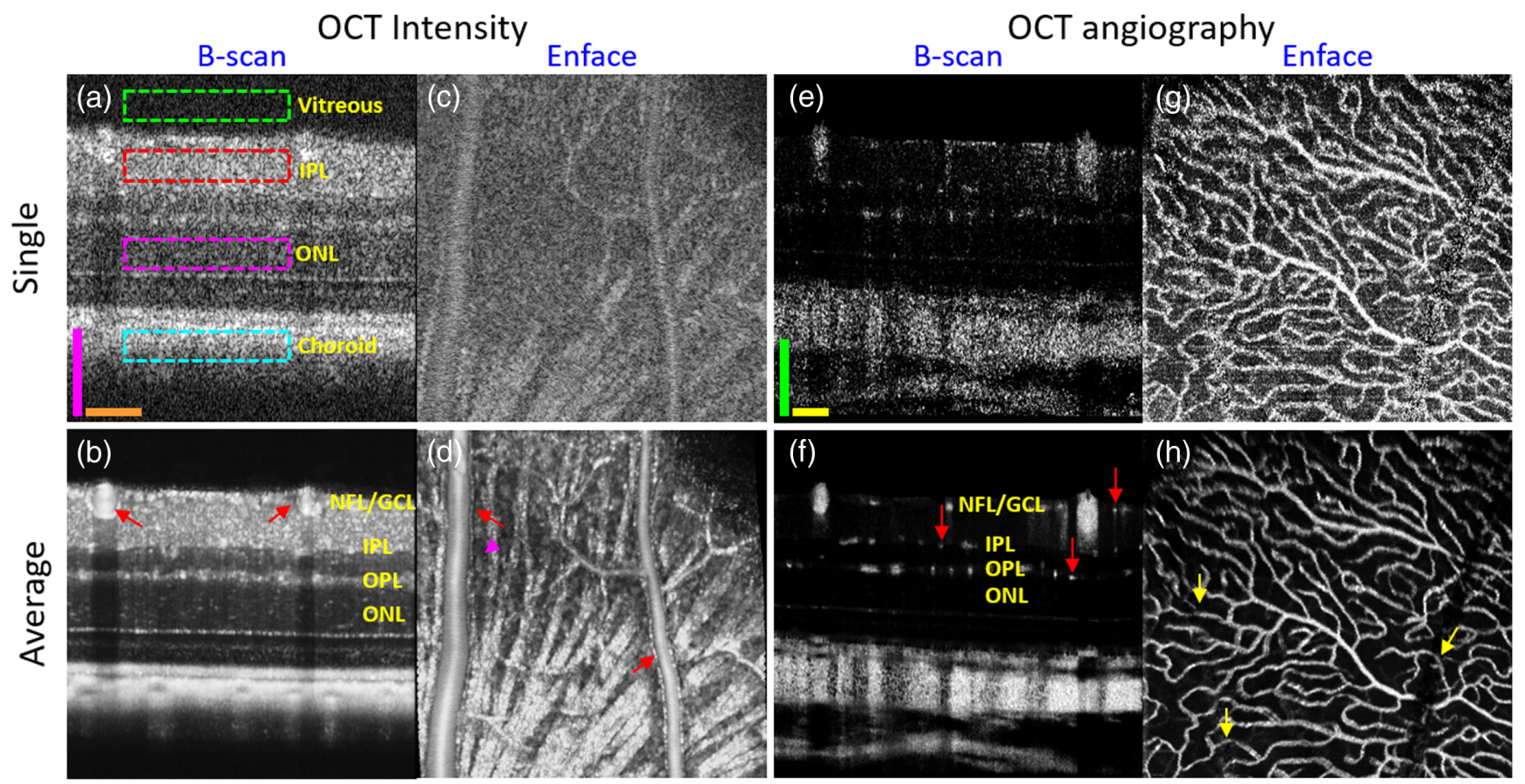

Fig. 4 Comparison of images from single and TSA OCT/OCTA volumes: (a) and (c) individual B-scan and en face images from a single volume; (b) and (d) individual B-scan and en face images from a TSA OCT volume reconstructed from 150 serially acquired volumes; (e) and (g) individual $B$-scan and en face OPL images from single OCTA volume; (f) and (h) B-scan and en face images from a TSA OCTA volume reconstructed from 50 serially acquired volumes. Scale bars: $100 \mu \mathrm{m}$. The en face images (c) and (d) were single layer (one axial pixel) images from the corresponding volumes; the en face images (g) and (h) were maximum projections from the layers around OPL (50 axial pixels). Abbreviations: NFL/GCL, nerve fiber layer/ganglion cell layer; IPL, inner plexiform layer; OPL, outer plexiform layer; ONL, outer nuclear layer.

averaging and SNR is clearly depicted by CNR [Fig. 5(c)], where after the initial reduction of speckle contrast and improvement of SNR for the first 50 volumes, the remaining improvement is mostly due to the reduction of speckle contrast. To further evaluate the change in speckle contrast, we estimated $m$ by fitting the full $K$-distribution [Eq. (4)] to the intensity histogram data from ROIs of TSA B-scans [Fig. 5(e)]. Figures 5(f) shows the estimation of uncorrelated speckle patterns in averaged B-scans implementing fitting of the full $K$-distribution to intensity histograms [Eq. (4)]. As expected, the number of uncorrelated speckle patterns increases steadily with $N$ but clearly deviates from the theoretical limit. The potential benefit of increasing the time interval between acquisition of consecutive volumes, what results in number of uncorrelated speckle fields, is also presented (square and star). Figure 5(f) also shows the comparison between independent speckle patters present in $N$-averaged volumes calculated using Eq. (3) for $M$ and Eq. (4) for $m$. It is evident that simple Eq. (3) underestimates the number of independent speckle patterns compared to results obtained with Eq. (4). This suggests the possible underestimation of the CNR.

\subsection{Example Results of TSA of OCT and OCTA}

To explore the effect of TSA on the visualization of neuronal and vascular structures in mouse retinal OCT data sets, we applied our technique to imaging pigmented C57BL/6 and B6 albino mice, which are two commonly used mouse genotypes. The C57BL/6 mouse line is one of the most popular mouse strains used in biomedical research. Addition of B6-albino mice allows the investigation of the effect of melanin on retinal data acquired with TSA OCT. Lack of melanin allows the imaging light to penetrate deeper into the choroid in albino mouse, which makes the imaging of choroidal vasculature less challenging than it is in pigmented C57BL/6 mice.

\subsubsection{TSA of 150 intensity volumes from a C57BL/6 mouse retina}

To further explore the value of TSA in retinal imaging, in Fig. 6, we present the results from applying TSA to 150 serially collected OCT volumes from a region of the retina of a pigmented (C75BL/6) mouse spanning a $600 \times 600 \mu \mathrm{m}^{2}$ and sampled by $512 \times 512$ pixels. The OCT beam was focused on the superficial retinal layers to better visualize inner retina. Figure 6 shows the results from seven representative layers extracted from the TSA OCT volume, clearly illustrating the improved visibility and contrast. Specifically, the nerve fiber bundles in the NFL, the blood vessels and capillaries in NFL, inner plexiform layer (IPL), and OPL are clearly visible. In addition, one can identify bright circular objects in ONL whose size appears to match the size of the photoreceptor cell bodies by comparing the OCT image with histology data ${ }^{45}$ [Fig. 6(f) zoom-in inset], microglia-like structures on top of the retina [Fig. 6(b) arrows. Inset shows an example of retinal SLO fluorescence image with green fluorescent protein (GFP)-labeled microglia acquired at the same scale, where several microglia cell bodies and corresponding processes are visible], and the cell soma-like structures 

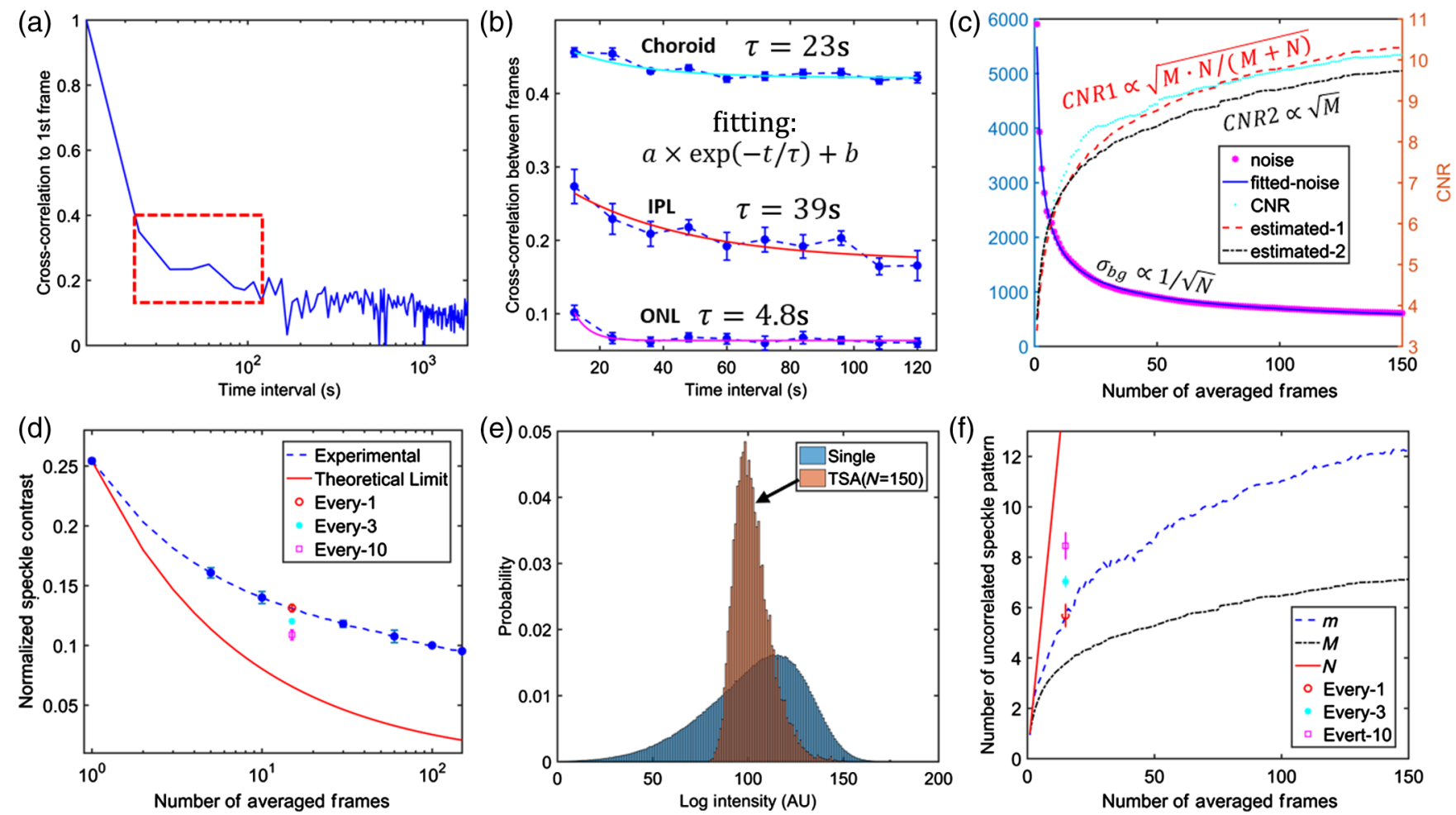

Fig. 5 Quantification of the effect of TSA by using the OCT intensity data from Fig. 4: (a) cross correlation between the first B-scan and consecutive B-scans from a single BM stack; (b) averaged cross correlation between B-scans from three different locations in a BM stack separated by different time intervals [the range depicted by the rectangle in (a), and the locations were marked by the corresponding dashed rectangles in Fig. 4(a)]; (c) noise and CNR [calculated from data obtained from the regions depicted by the top two rectangles in Fig. 4(a)] as a function of the number $N$ of averaged volumes; (d) NSC changes calculated for the IPL rectangle in Fig. 4(a) as a function of the number of averaged volumes; (e) example intensity histogram for single and TSA images from the data in the IPL rectangle in Fig. 4(a); (f) estimated number of uncorrelated speckle patterns after averaging shown as a function of the number of averaged volumes $N$.
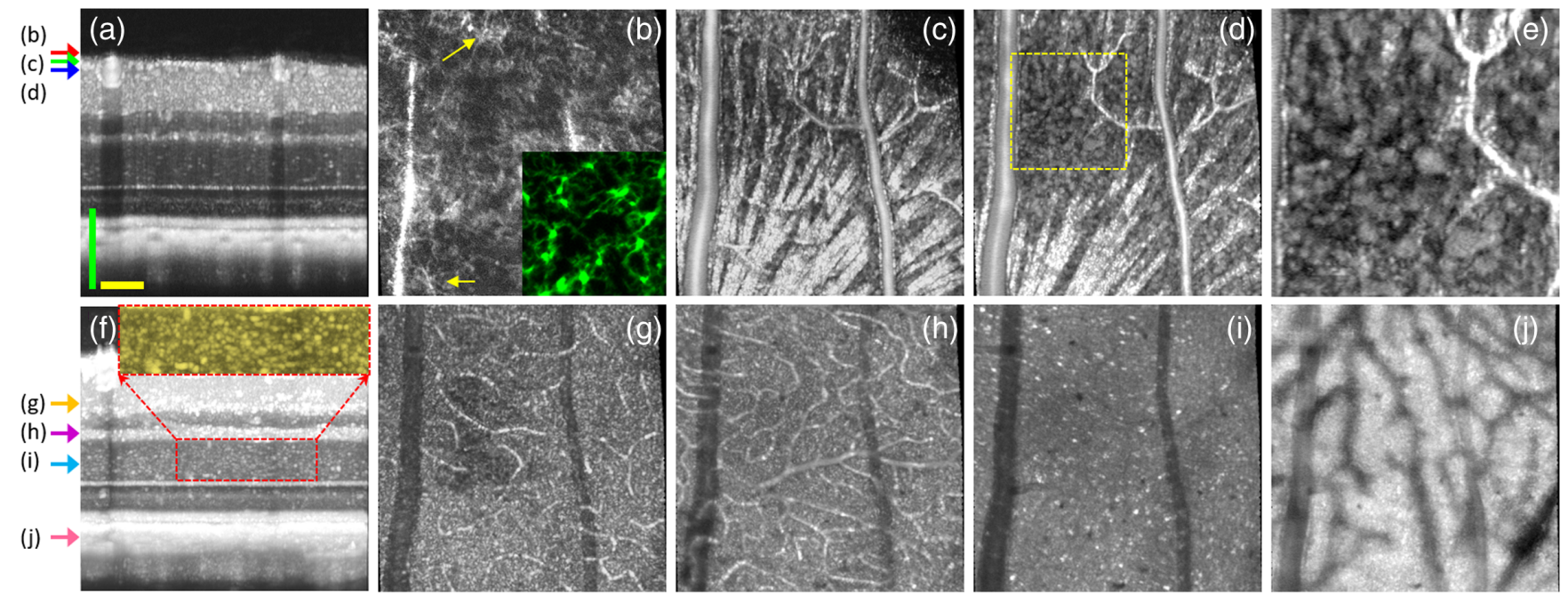

Fig. 6 Visualization of a TSA volume from a 150 serially acquired OCT volumes from a pigmented (C57BI/6J) mouse. (a) the central B-scan from the TSA volume; (b)-(d) three representative en face images extracted from the volume with depth $(z)$ locations indicated by the correspondingly arrows in (a); (e) a magnified depiction of the region in (d) indicated by the dashed square; (b) inset: example fluorescence image of GFP-labeled microglia cells; (f) the "Max Intensity" B-scan projection of the entire TSA volume, inset: zoom-in image of the structures in ONL; (g)-(j) four representative en face images extracted from the TSA volume with depth locations indicated by the corresponding arrows in (f). Scale bars: $100 \mu \mathrm{m}$. 
in the ganglion cell layer (GCL) [Figs. 6(d)-6(e)]. The putative cell somas in the GCL could belong to GCs and/or amacrine cells, and the specific cellular origin of these structures needs to be further tested. The ability to observe cell somas opens the door to quantitative assessment of several retina cell types in vivo, going beyond the classic measurement of retina layer thickness commonly used in the presentation of OCT results till date. Together, these results provide a first glimpse of the improvement in the visualization of cellular morphology achievable by the application of TSA to OCT volumes.

\subsubsection{TSA of 50 OCTA volumes from a B6 albino mouse retina}

OCTA is a powerful technique that allows the visualization of blood vessels without an extrinsic contrast agent. ${ }^{25,32}$ Here we illustrate the application of TSA to serially acquired OCTA volumes. As mentioned above, one of the features of our volume registration method (Fig. 2) is that it allows multichannel registration based on the structural (intensity) OCT data alone. Here we demonstrate this multimodal functionality by coregistering 50 OCTA volumes acquired over $1 \times 1 \mathrm{~mm}^{2}$ with $540 \times 1080$ $(360 \times 3)$ A-scans (Fig. 7). In this data acquisition protocol, three B-scans are collected consecutively at each $y$ position to extract the phase variance that arises from the blood cells scattering as they transit the vasculature. ${ }^{32,33}$ For this experiment, a B6 albino mouse was imaged, and the optical focus of the system was set in the middle of the retina near the ONL to achieve good quality imaging of both the retinal and the choroidal vasculature. Similar to the previous example (Fig. 6), large structures, including blood vessels and nerve fiber bundles, are visualized with very good quality by the TSA OCT intensity data. The cell somas in the GCL [Fig. 7(c)] are barely visible due to the shift in the optical focus away from the GCL and lower spatial sampling from the wider PSF. The TSA OCTA volume offers clear visualization of the choroid [Fig. 7(f)] and, more strikingly, of the choriocapillaris [Fig. 7(g)], which has not been previously visualized in the mouse in vivo without contrast agent. ${ }^{46,47}$ OCTA data sets can be used to produce very high contrast maps of retinal vasculature, as illustrated with a depthcolor-coded projection of the inner retina vessels [Fig. 7(h)].

\section{Discussion and Conclusions}

In this paper, we presented a framework for applying TSA to cellular resolution volumetric neuron and vascular imaging of the living mouse retina. Successful visualization of novel cellular structures was achieved by the application of a stepwise registration method (Fig. 2). The method was built on reliable and robust registration tools available in ImageJ, the common open-source data-processing platform developed by $\mathrm{NIH}^{23}$ As a result, the proposed method is simple to adopt if compared to dedicated custom registration, which may lower some technical barriers to its adoption. Although the examples shown in this paper use data from mouse retinal imaging, we believe that this method can be easily extended to OCT volumes acquired in any anesthetized subjects, including other experimental animals and humans (e.g., infants) (the eye movements of nonanesthetized subjects present additional challenges). In addition, our method allows extraction of the retina motions present during imaging, which might help to troubleshoot sources of unwanted motion in serially acquired volumes.

In addition to the application of TSA to OCT intensity data sets (Fig. 6), we also showcased TSA of OCTA volumes (Fig. 7). In this case, TSA provided high contrast for retinal vasculature and choroid. To the best of our knowledge, TSA yielded the first contrast-free visualization of the mouse choriocapillaris. The importance of noninvasive choriocapillaris visualization has been pointed out by clinical studies in humans, ${ }^{48-51}$ and the general understanding that this distinctive vascular bed supplies nutrition and oxygen to the retinal pigment epithelium (RPE)
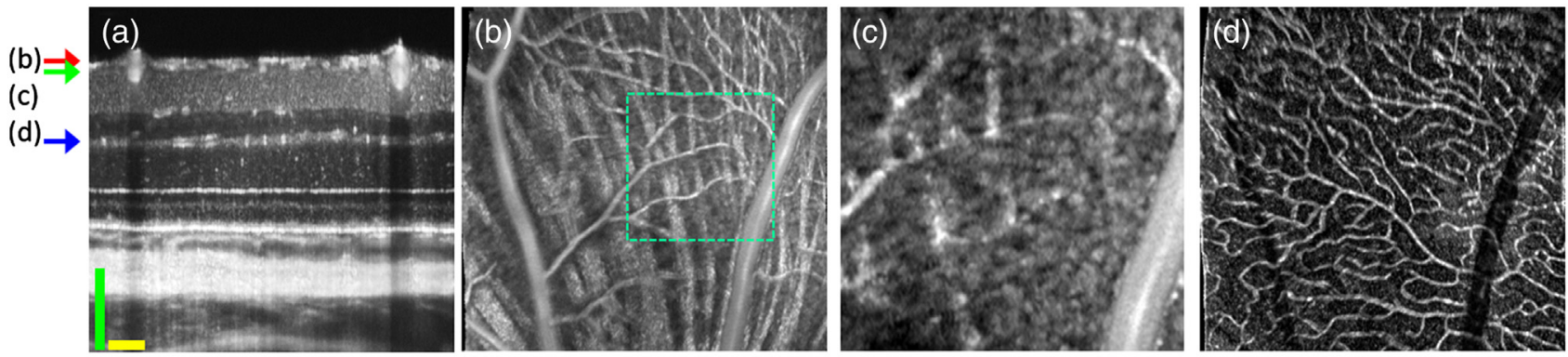

(e)
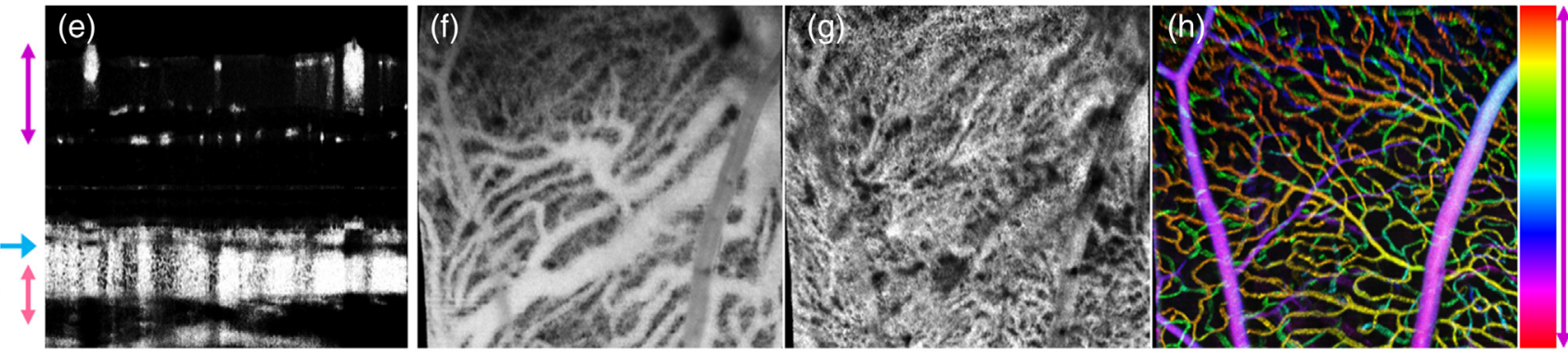

Fig. 7 Results from the application of TSA to data created from 50 serially acquired OCTA volumes: (a) the central B-scan from the TSA OCT intensity volume; (b)-(d) three representative en face images extracted from the averaged volume with depth $(z)$ locations indicated by the correspondingly arrows in (a), and the lateral location of (c) indicated by the green rectangle in (b); (e) the central B-scan of the TSA OCTA volume; (f)-(h) three representative en face projections from the TSA OCTA volume with axial location indicated by the corresponding arrows in (e). Scale bars: $100 \mu \mathrm{m}$. 
and photoreceptors, ${ }^{52}$ and that its deterioration may play a key role in the development of many retina diseases including agerelated macular degeneration (AMD). ${ }^{52}$ TSA OCTA should provide improved visualization of neovascularization, an important metric for monitoring the progress of AMD toward the "wet" form, and for ocular tumors. ${ }^{53}$

TSA greatly improves the image quality by two distinctive but overlapping statistical mechanisms. The first is the general process by which averaging reduces noise-which in general may arise from shot-noise fluctuations in photon capture rates or instrumental noise-while simultaneously increasing the dynamic range of the signal. However, in the case of Fourierdomain OCT detection that is used in acquiring our volumetric data sets, we know that the shot-noise is limiting. ${ }^{54-56}$ This process enables structures that weakly scatter to become visible. Without TSA, however, sample-to-sample distortions arising from ocular motion can reduce resolution and reduce the effectiveness of averaging.

The second mechanism by which TSA improves image quality is by suppression of speckle contrast. It is increasingly clear that many of the sub-PSF scale scatterers that produce speckle in imaging with partially coherent light are mobile. TSA reduces speckle contrast because of the time-dependent decorrelation of the speckle patterns in the living retina (Fig. 5). Investigation of the decorrelation time for speckle in different cellular and subcellular locations may provide information about the identity and mobility of the underlying scatterers. ${ }^{57-59}$ While decorrelation times are set by the biological properties of the underlying scatterers, when considered purely as noise, it is possible to speed decorrelation by subtle manipulations of the PSF, as we described recently in an active method that creates uncorrelated speckle patterns with aperture phase modulation (APM) via AO-OCT. ${ }^{45}$ This latter technique can more efficiently reduce speckle contrast since it need not wait on the biological decorrelation time but does so at the cost of the increased complexity of the imaging system. The TSA method needs no additional components beyond those available with any mouse retinal OCT system. It is important to mention that decorrelation time for speckle patterns acquired in vivo in clinical AO-OCT systems (NFL, GCL, IPL $<1 \mathrm{~s},{ }^{58} \mathrm{RPE}<3 \mathrm{~s}^{60}$ ) could be shorter than the one reported in this paper $(\tau=5$ to $40 \mathrm{~s})$. There can be several reasons besides biological difference between species that could explain this discrepancy, including: (1) difference in pupil stability between awake human and anesthetize mouse. Our recent study on the influence of ocular aberrations on speckle patterns suggests that variations in pupil wavefront during clinical imaging could result in unintended aperture phase modulations, ${ }^{45}$ which could affect speckle decorrelation times. (2) Lateral and axial resolution between OCT and AOOCT system. Narrower PSFs should be more sensitive to local variations in scattering, resulting in shorter decorrelation time. (3) The speckle decorrelation times reported in this study may be affected by the relatively slow volume acquisition rates. Clearly, more studies are needed to resolve these differences and weight influence of each factor.

One of the limitations of the registration method as currently implemented is its relatively long processing time, 3 to $4 \mathrm{~h}$ for a 50 -volume data set. Even though the analysis is mostly automated, the lengthy processing time limits the ability to perform this analysis routinely. The long processing time is a consequence of using open source tools developed for 2-D image registration. Thus, there is a need for a dedicated graphics processing-unit-based image registration process that could, for example, perform parallel registrations of BM stacks. This would greatly reduce the processing time, opening the door to iterative registration that would further reduce motion artifacts.

The data in this paper were acquired using a non-AO-OCT mouse retina system. We expect that this framework can be extended to AO-OCT data acquired over smaller fields of view, usually a few degrees of the visual field, as long as the serial en face intensity projection and B-scans can be registered using the ImageJ plugins. Our retinal neuron soma images have lower contrast than those from human retina acquired with AOOCT. ${ }^{16,20}$ This lower contrast could be because the NA of the mouse retina imaging system used in this paper is only one-half of that used in the human system. In future, we will apply TSA to data sets acquired by a compact AO-OCT system $^{45}$ which has an NA of 0.23 , roughly equal to the human full-pupil AO-OCT system. This increased NA will improve lateral resolution, and potentially, the cellular contrast in our data. However, the ability to visualize the highly transparent somas of the neurons in the mouse retina without the use of AO makes the TSA very attractive. As an alternative to AO, it is possible to use a somewhat larger imaging beam size on the mouse pupil to increase the system NA, ${ }^{29}$ or switch to shorter wavelength (visible light $\mathrm{OCT}^{61,62}$ ) imaging. Both strategies will predictably improve the system resolution and yield a better contrast of cell somas. Finally, other promising alternatives to hardware-based $\mathrm{AO}$, such as computational AO techniques,${ }^{63}$ could also be combined with our method to provide high-quality retinal images.

\section{Disclosures}

All authors declare no conflicts of interest.

\section{Acknowledgments}

Authors would like to acknowledge their funding sources: UC Davis; NSF I/UCRC CBSS Grant; National Institutes of Health Grant Nos. EY026556, EY02660, and EY012576 (NEI Core Grant); Vision Science T32 EY015387; and UC Davis Travel Grant No. S-PZ07427. We thank Professor Marinko V. Sarunic of Simon Fraser University and Professor Yifan Jian of Oregon Health and Science University for their ongoing help and discussions and for sharing MATLAB code for initial OCT and OCTA data processing. We also thank Professor Vivek Jay Srinivasan for sharing the real-time OCT processing DLL; we thank Professors Marie Burns and John S. Werner for their help and support. We thank Dr. Robert H. Cudmore for his input on MultiStackReg plugin.

\section{References}

1. G. Söhl et al., "The murine gap junction gene connexin36 is highly expressed in mouse retina and regulated during brain development," FEBS Lett. 428(1-2), 27-31 (1998).

2. A. London, I. Benhar, and M. Schwartz, "The retina as a window to the brain-from eye research to CNS disorders," Nat. Rev. Neurol. 9, 44-53 (2012).

3. J. Z. Kieval et al., "Ultra-high resolution optical coherence tomography for differentiation of ocular surface squamous neoplasia and pterygia," Ophthalmology 119(3), 481-486 (2012).

4. C. J. Bult et al., "The Mouse Genome Database (MGD): mouse biology and model systems," Nucleic Acids Res. 36(Suppl. 1), D724-D728 (2008).

5. B. Chang et al., "Retinal degeneration mutants in the mouse," Vision Res. 42(4), 517-525 (2002). 
6. G. J. Chader, "Animal models in research on retinal degenerations: past progress and future hope," Vision Res. 42(4), 393-399 (2002).

7. P. Zhang et al., "In vivo optophysiology reveals that G-protein activation triggers osmotic swelling and increased light scattering of rod photoreceptors," Proc. Natl. Acad. Sci. U. S. A. 114(14), E2937-E2946 (2017).

8. B. Ellenbroek and J. Youn, "Rodent models in neuroscience research: is it a rat race?" Dis. Model Mech. 9(10), 1079-1087 (2016).

9. Y. Geng et al., "Optical properties of the mouse eye," Biomed. Opt. Express 2(4), 717-738 (2011).

10. Y. Geng et al., "Adaptive optics retinal imaging in the living mouse eye," Biomed. Opt. Express 3(4), 715-734 (2012).

11. R. H. Webb and G. W. Hughes, "Scanning laser ophthalmoscope," IEEE Trans. Biomed. Eng. BME-28(7), 488-492 (1981).

12. D. Huang et al., "Optical coherence tomography," Science 254(5035), 1178-1181 (1991).

13. P. Zhang et al., "In vivo wide-field multispectral scanning laser ophthalmoscopy-optical coherence tomography mouse retinal imager: longitudinal imaging of ganglion cells, microglia, and Müller glia, and mapping of the mouse retinal and choroidal vasculature," J. Biomed. Opt. 20, 126005 (2015).

14. D. J. Wahl et al., "Sensorless adaptive optics multimodal en-face small animal retinal imaging," Biomed. Opt. Express 10(1), 252-267 (2019).

15. R. J. Zawadzki et al., "Adaptive-optics optical coherence tomography for high-resolution and high-speed 3D retinal in vivo imaging," Opt. Express 13(21), 8532-8546 (2005).

16. Z. Liu, J. Tam, and O. Saeedi et al., "Trans-retinal cellular imaging with multimodal adaptive optics," Biomed. Opt. Express 9(9), 4246-4262 (2018).

17. J. Porter et al., Adaptive Optics for Vision Science, John Wiley \& Sons, Inc., Hoboken, New Jersey (2006).

18. R. J. Zawadzki et al., "Adaptive-optics SLO imaging combined with widefield OCT and SLO enables precise 3D localization of fluorescent cells in the mouse retina," Biomed. Opt. Express 6(6), 2191-2210 (2015).

19. E. A. Rossi et al., "Imaging individual neurons in the retinal ganglion cell layer of the living eye," Proc. Natl. Acad. Sci. U. S. A. 114(3), 586-591 (2017).

20. Z. Liu et al., "Imaging and quantifying ganglion cells and other transparent neurons in the living human retina," Proc. Natl. Acad. Sci. U. S. A. 114(48), 12803-12808 (2017).

21. E. M. Wells-Gray et al., "Inner retinal changes in primary open-angle glaucoma revealed through adaptive optics-optical coherence tomography," J. Glaucoma 27(11), 1025-1028 (2018).

22. A. Guevara-Torres, D. R. Williams, and J. B. Schallek, "Imaging translucent cell bodies in the living mouse retina without contrast agents," Biomed. Opt. Express 6(6), 2106-2119 (2015).

23. C. A. Schneider, W. S. Rasband, and K. W. Eliceiri, "NIH image to ImageJ: 25 years of image analysis," Nat. Methods, 9, 671-675 (2012).

24. I. Gorczynska et al., "Comparison of amplitude-decorrelation, specklevariance and phase-variance OCT angiography methods for imaging the human retina and choroid," Biomed. Opt. Express 7(3), 911-942 (2016).

25. Y. Jia et al., "Quantitative optical coherence tomography angiography of vascular abnormalities in the living human eye," Proc. Natl. Acad. Sci. U. S. A. 112(18), E2395-E2402 (2015).

26. P. Zhang et al., "Effect of a contact lens on mouse retinal in vivo imaging: Effective focal length changes and monochromatic aberrations," Exp. Eye Res. 172, 86-93 (2018).

27. R. W. Ditchburn and B. L. Ginsborg, "Involuntary eye movements during fixation," J. Physiol. 119(1), 1-17 (1953).

28. P. Zhang et al., "The photosensitivity of rhodopsin bleaching and lightinduced increases of fundus reflectance in mice measured in vivo with scanning laser ophthalmoscopy," Invest. Ophthalmol. Visual Sci. 57(8), 3650-3664 (2016).

29. P. Zhang et al., "Effect of scanning beam size on the lateral resolution of mouse retinal imaging with SLO," Opt. Lett. 40(24), 5830-5833 (2015).

30. Y. Jian, K. Wong, and M. V. Sarunic, "Graphics processing unit accelerated optical coherence tomography processing at megahertz axial scan rate and high resolution video rate volumetric rendering," J. Biomed. Opt. 18(2), 026002 (2013).

31. W. D. A.F. Fercher, C. K. Hitzenberger, and T. Lasser, "Optical coherence tomography-principles and applications," Rep. Prog. Phys. 66, 239-303 (2003).
32. D. Y. Kim et al., "Optical imaging of the chorioretinal vasculature in the living human eye," Proc. Natl. Acad. Sci. U. S. A. 110(35), 14354-14359 (2013).

33. D. Y. Kim et al., "In vivo volumetric imaging of human retinal circulation with phase-variance optical coherence tomography," Biomed. Opt. Express 2(6), 1504-1513 (2011).

34. R. J. Zawadzki et al., "Adaptive optics-optical coherence tomography: optimizing visualization of microscopic retinal structures in three dimensions," J. Opt. Soc. Am. A 24(5), 1373-1383 (2007).

35. P. Thevenaz, U. E. Ruttimann, and M. Unser, "A pyramid approach to subpixel registration based on intensity," IEEE Trans. Image Process. 7(1), 27-41 (1998).

36. B. Busse, "MultiStackReg v1.45," http://bradbusse.net/sciencedownloads .html.

37. T. M. Jørgensen et al., "Enhancing the signal-to-noise ratio in ophthalmic optical coherence tomography by image registration —-method and clinical examples," J. Biomed. Opt. 12(4), 041208 (2007).

38. R. J. Zawadzki et al., "Ultrahigh-resolution optical coherence tomography with monochromatic and chromatic aberration correction," Opt. Express 16(11), 8126-8143 (2008).

39. X. Song et al., "Automated region detection based on the contrast-tonoise ratio in near-infrared tomography," Appl. Opt. 43(5), 1053-1062 (2004).

40. J. W. Goodman, "Some fundamental properties of speckle," J. Opt. Soc. Am. 66(11), 1145-1150 (1976).

41. J. M. Schmitt, S. H. Xiang, and K. M. Yung, "Speckle in optical coherence tomography," J. Biomed. Opt. 4(1), 95-105 (1999).

42. T. B. Dubose et al., "Statistical models of signal and noise and fundamental limits of segmentation accuracy in retinal optical coherence tomography," IEEE Trans. Med. Imaging 37(9), 1978-1988 (2018).

43. M. Sugita et al., "Analysis of scattering statistics and governing distribution functions in optical coherence tomography," Biomed. Opt. Express 7(7), 2551-2564 (2016).

44. Z. Liu, O. P. Kocaoglu, and D. T. Miller, "3D imaging of retinal pigment epithelial cells in the living human retina3D imaging of RPE cells in living human retina," Invest. Ophthalmol. Visual Sci. 57(9), OCT533-OCT543 (2016).

45. P. Zhang et al., "Aperture phase modulation with adaptive optics: a novel approach for speckle reduction and structure extraction in optical coherence tomography," Biomed. Opt. Express 10(2), 552-570 (2019).

46. M. Goswami et al., "Visualization of chorioretinal vasculature in mice in vivo using a combined OCT/SLO imaging system," Proc. SPIE 9693 , 96930G (2016).

47. M. T. Bernucci, C. W. Merkle, and V. J. Srinivasan, "Investigation of artifacts in retinal and choroidal OCT angiography with a contrast agent," Biomed. Opt. Express 9(3), 1020-1040 (2018).

48. J. V. Migacz et al., "Megahertz-rate optical coherence tomography angiography improves the contrast of the choriocapillaris and choroid in human retinal imaging," Biomed. Opt. Express 10(1), 50-65 (2019).

49. B. Braaf et al., "Angiography of the retina and the choroid with phaseresolved OCT using interval-optimized backstitched B-scans," Opt. Express 20(18), 20516-20534 (2012).

50. W. Choi et al., "Choriocapillaris and choroidal microvasculature imaging with ultrahigh Speed OCT angiography," PLoS One, 8(12), e81499 (2013).

51. K. Kurokawa, Z. Liu, and D. T. Miller, "Adaptive optics optical coherence tomography angiography for morphometric analysis of choriocapillaris [invited]," Biomed. Opt. Express 8(3), 1803-1822 (2017).

52. I. Bhutto and G. Lutty, "Understanding age-related macular degeneration (AMD): relationships between the photoreceptor/retinal pigment epithelium/Bruch's membrane/choriocapillaris complex," Mol. Aspects Med. 33(4), 295-317 (2012).

53. M. Goswami et al., "Novel window for cancer nanotheranostics: noninvasive ocular assessments of tumor growth and nanotherapeutic treatment efficacy in vivo," Biomed. Opt. Express 10(1), 151-166 (2019).

54. J. F. de Boer et al., "Improved signal-to-noise ratio in spectral-domain compared with time-domain optical coherence tomography," Opt. Lett. 28(21), 2067-2069 (2003).

55. R. Leitgeb, C. K. Hitzenberger, and A. F. Fercher, "Performance of Fourier domain vs. time domain optical coherence tomography," Opt. Express 11(8), 889-894 (2003). 
56. M. A. Choma et al., "Sensitivity advantage of swept source and Fourier domain optical coherence tomography," Opt. Express 11(18), 2183-2189 (2003).

57. O. Thouvenin et al., "Cell motility as contrast agent in retinal explant imaging with full-field optical coherence tomography," Invest. Ophthalmol. Visual Sci. 58(11), 4605-4615 (2017).

58. K. Kurokawa et al., "Method to investigate temporal dynamics of ganglion and other retinal cells in the living human eye," Proc. SPIE 10474, 104740W (2018).

59. J. Scholler et al., "Probing dynamic processes in the eye at multiple spatial and temporal scales with multimodal full field OCT," Biomed. Opt. Express 10(2), 731-746 (2019).

60. Z. Liu et al., "Characterizing motility dynamics in human RPE cells," Proc. SPIE 10045, 1004515 (2017).

61. S. P. Chong et al., "Structural and functional human retinal imaging with a fiber-based visible light OCT ophthalmoscope," Biomed. Opt. Express 8(1), 323-337 (2017).

62. J. Yi et al., "Visible-light optical coherence tomography for retinal oximetry," Opt. Lett. 38(11), 1796-1798 (2013).

63. S. G. Adie et al., "Computational adaptive optics for broadband optical interferometric tomography of biological tissue," Proc. Natl. Acad. Sci. U. S. A. 109(19), 7175-7180 (2012).

Pengfei Zhang is a project scientist working at the University of California Davis (UC Davis). His research focuses on developing advanced optical imaging instruments and tools for in vivo small animal retina structural and functional imaging.
Eric B. Miller is a PhD candidate at Center for Neuroscience in UC Davis. His research interests include the impact of retina light damage on microglia and immune cells, using both in vivo and ex vivo tools.

Suman K. Manna is a postdoc at UC Davis. His research subject is to study retinal tumor in mouse model: monitoring the tumor growth in vivo and testing different drugs and therapies to cure the tumor.

Ratheesh K. Meleppat is a postdoc at UC Davis. His primary research is developing in-vivo imaging tools to study the directional optical signals in relation to the melanin concentration-dependent scattering properties of retinal pigment epithelium.

Edward N. Pugh, Jr. is a professor at UC Davis in the Departments of Cell Biology and Human Anatomy and the Department of Physiology and Membrane Biology. His research program has for many years investigated phototransduction, the molecular and biophysical mechanisms by which rod and cone photoreceptors in the retina transduce light into electrical signals.

Robert J. Zawadzki is an associate professor in the Department of Ophthalmology in UC Davis. His research interests focus on development of new instrumentation for high-resolution in-vivo retina imaging (allowing visualization of individual cellular structures). This includes but is not limited to optical coherence tomography, scanning laser ophthalmoscopy, adaptive optics, and combinations of all the above. 